\title{
The Theodorus Variation
}

\author{
EWAN BRINKMAN, Simon Fraser University, Canada \\ ROBERT M. CORLESS, Western University, Canada \\ VESELIN JUNGIĆ, Simon Fraser University, Canada
}

\section{Recommended Reference Format:}

Ewan Brinkman, Robert M. Corless, and Veselin Jungić. 2021. The Theodorus Variation. Maple Trans. 1, 2, Article 14500 ( 2021), 17 pages. https://doi.org/10.5206/mt.v1i2.14500

\section{Introduction}

In this note we study what Susan Gerofsky, a Canadian mathematics educator, in [10] calls the reverse Wurzelschnecke. "Wurzelschnecke" is German for "root snail"; this will make sense very soon. This object, this "root snail backward," is closely related to the Spiral of Theodorus, an ancient figure recently given that name in [8, p. 33], that still attracts attention of mathematicians [9] and artists [15] alike.

To construct the Spiral of Theodorus, Figure 2, we start with the equilateral right triangle $\triangle \mathbf{O T}_{1} \mathbf{T}_{2}$ with $\left|\overline{\mathrm{OT}_{1}}\right|=\left|\overline{\mathrm{T}_{1} \mathrm{~T}_{2}}\right|=1$. The hypotenuse is therefore of length $\sqrt{2}$. The point $\mathrm{T}_{n}$, for $n \geq 3$, is determined so that $\angle \mathrm{OT}_{n-1} \mathbf{T}_{n}$ is the right angle and that $\left|\overline{\mathbf{T}_{n-1} \mathbf{T}_{n}}\right|=1$. The side lengths of $\triangle \mathbf{O T} \mathbf{T}_{n-1} \mathbf{T}_{n}$ are $\sqrt{n-1}, 1$, and $\sqrt{n}=\sqrt{(\sqrt{n-1})^{2}+1^{2}}$. See Figure 1 . Observe that the spiral in Figure 2 is (arbitrarily) oriented counterclockwise.

It is a straightforward observation ${ }^{1}$ that $\left|\overline{\mathrm{OT}_{n}}\right|=\sqrt{n}$. This can be proved by mathematical induction and the Pythagorean theorem, for instance. This is the reason that the Spiral of Theodorus is sometimes called the "square root spiral."

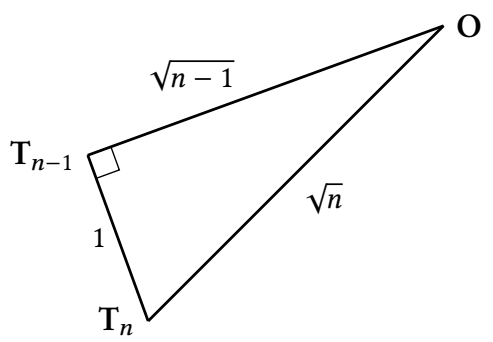

Fig. 1. The right triangle $\Delta \mathrm{OT}_{n-1} \mathrm{~T}_{n}$, which has one side of unit length, and the others of length $\sqrt{n-1}$ and $\sqrt{n}$. Pythagoras' theorem is seen to hold: $(\sqrt{n-1})^{2}+(1)^{2}=(\sqrt{n})^{2}$.

\footnotetext{
${ }^{1}$ That is, straightforward for practiced geometers and other mathematicians. The point of doing this with elementary students who may just be learning the Pythagorean theorem is to get them to appreciate this fact. Even to get the students to appreciate that the shorthand $\sqrt{n}$ applies to all of the triangles in turn, starting from $n=2, n=3$, and so on, is important.

Authors' addresses: Ewan Brinkman, ewanbrinkman@gmail.com, Simon Fraser University, Burnaby, British Columbia, Canada, V5A 1S6; Robert M. Corless, rcorless@uwo.ca, Western University, London, Ontario, Canada, N6A 5B7; Veselin Jungić, vjungic@sfu.ca, Simon Fraser University, Burnaby, British Columbia, Canada, V5A 1S6.

Permission to make digital or hard copies of all or part of this work for any use is granted without fee, provided that copies bear this notice and the full citation on the first page. Copyrights for third-party components of this work must be honored (c) 2021 Copyright held by the owner/author(s). Publication rights licensed to Maple Transactions, under Creative Commons CC-BY 4.0 License.

https://doi.org/10.5206/mt.v1i2.14500
} 


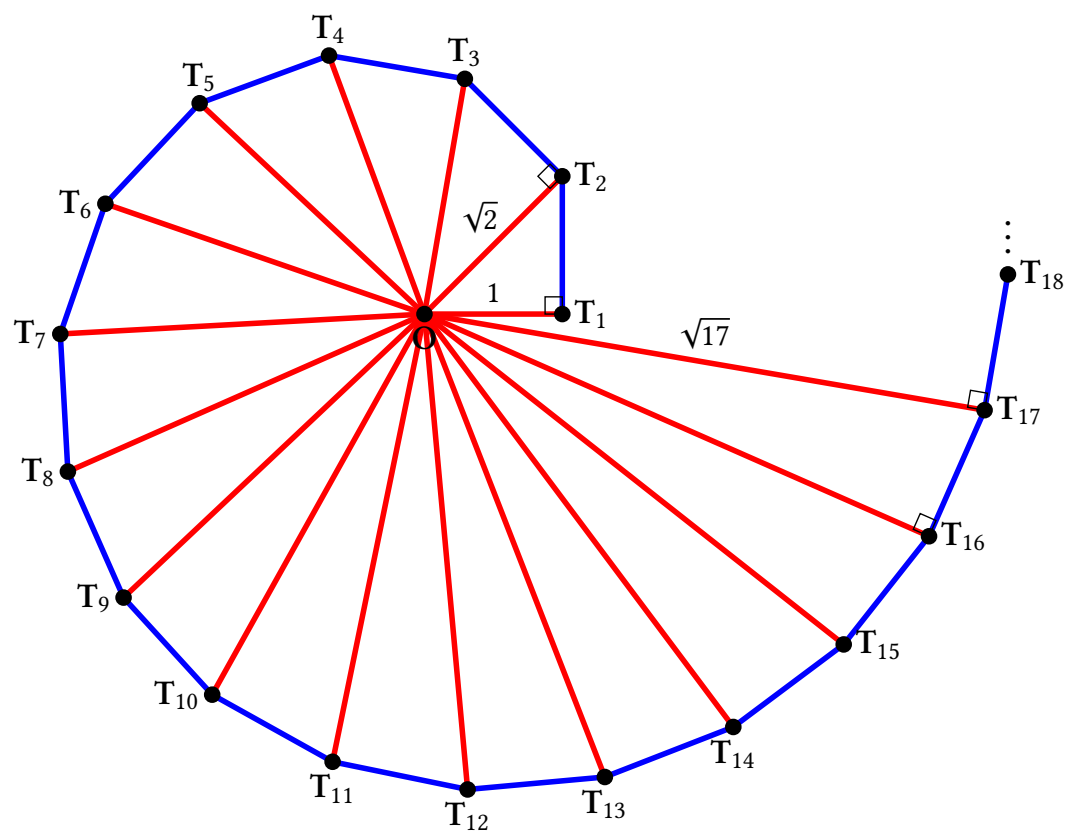

Fig. 2. The Spiral of Theodorus, built by placing the basic right triangles with the base of each subsequent triangle on the hypotenuse of the previous triangle. By construction, these are the same length.

Theodorus of Cyrene [14] lived in the fifth century BCE and was "mentioned by Proclus along with Hippocrates as a celebrated geometer." [12, p. 202]

Sir Thomas Heath (1861 -1940), a British classicist, in his book A History of Greek Mathematics writes:

In Plato's Theaetetus we have the story of Theodorus lecturing on surds and proving separately, for the square root of every non square number from 3 to 17 , that it is incommensurable with 1. [12, p. 22]

Later in the text, Heath explains:

Plato gives no hint as to how Theodorus proved the propositions attributed to him, namely that $\sqrt{3}, \sqrt{5}, \ldots, \sqrt{17}$ are all incommensurable with 1 ; there is therefore a wide field open for speculation, and several conjectures have been put forward. [12, p. 204]

The modern reader is probably familiar with the argument that the identity $p^{2}=n \cdot q^{2}$, for $n, p$ and $q$ being integers, implies, from unique factorization, that every prime divisor of $n$ appears to an even power, and hence $n$ is a perfect square.

We observe that this "modern" proof is practically the same reductio ad absurdum argument that Pythagoreans used to prove that $\sqrt{2}$ is irrational. Keeping in mind that Theodorus's proof appeared several decades after the discovery of the irrationality of $\sqrt{2}$, it is a natural question to ask about the value of Theodorus's approach.

Heath discusses this question in some length by presenting a few hypothesis about the nature of Theodorus's proof. One of those hypotheses, that Heath attributes to Friedrich Hultsch (1833 1906), a German classical philologist, was that Theodorus actually, to use a modern term, visualized Pythagoreans' reductio ad absurdum argument. [12, pp. $204-205]$ 
Fast forward to the 21st century, Jonathan Borwein (1953 - 2016), a Canadian mathematician, as an example of experimental mathematic accessible at the high school level, in multiple publications used an image that depicts the fact that an assumption that $\sqrt{2}$ is rational leads to a contradiction. See, for example, $[2,4,1,3]$.

In the spirit of experimental mathematics ${ }^{2}$, Borwein (and his coauthor) advise the reader to basically go along the Spiral of Theodorus ${ }^{3}$ and create visual proof(s) that nonperfect squares do not have rational square roots.

For more about connections between the Spiral of Theodorus and a technique that Borwein called minimal configurations [3], the technique that emphasizes the visual side of the fact that the magnitudes $\sqrt{n}$, where $n \in \mathbb{N}$ is a nonperfect square, and 1 are incommensurable, see $[10,13]$.

\section{A continuous version}

Philip J. Davis (1923 - 2018), an American applied mathematician, has in [8, p. 38] the following, most mysterious, formula:

$$
T(a)=\prod_{k \geq 1} \frac{1+i / \sqrt{k}}{1+i / \sqrt{k+a-1}} .
$$

[Davis actually uses $a$ instead of $a-1$, there; we make this shift to accommodate the notation in this paper.] Here $i$ is the square root of -1 . This is an infinite product, but note that if the variable $a$ happens to be a positive integer, then something interesting happens, namely, most of the terms in the product cancel out. Let us do the computation explicitly if $a=4$. Note that the infinite product must be defined as a limit, say

$$
T(a)=\lim _{N \rightarrow \infty} \prod_{k=1}^{N} \frac{1+i / \sqrt{k}}{1+i / \sqrt{k+a-1}} .
$$

Letting $a=4$ and writing the first few terms out explicitly we have

$$
\begin{aligned}
T(4) & =\lim _{N \rightarrow \infty}\left(\frac{1+i / \sqrt{1}}{1+i / \sqrt{1+3}}\right)\left(\frac{1+i / \sqrt{2}}{1+i / \sqrt{2+3}}\right)\left(\frac{1+i / \sqrt{3}}{1+i / \sqrt{3+3}}\right)\left(\frac{1+i / \sqrt{4}}{1+i / \sqrt{4+3}}\right) \cdots\left(\frac{1+i / \sqrt{N}}{1+i / \sqrt{N+3}}\right) \\
& =\lim _{N \rightarrow \infty} \frac{(1+i / \sqrt{1})(1+i / \sqrt{2})(1+i / \sqrt{3})}{(1+i / \sqrt{N+1})(1+i / \sqrt{N+2})(1+i / \sqrt{N+3})},
\end{aligned}
$$

because all of the terms except the first 3 in the numerator and the last 3 in the denominator cancel, or "telescope." This limit can therefore be seen to exist, and equal $(1+i / \sqrt{1})(1+i / \sqrt{2})(1+i / \sqrt{3})$. A similar thing happens if $a$ is any positive integer; at the end, we are left with a finite product of complex numbers, and indeed $T(n+1)=(1+i / \sqrt{n}) T(n)$, and $T(1)=1$.

Great, you say. What has this to do with the spiral of Theodorus? The connection is as follows: embed the spiral in the complex plane, with the origin at $z=0+0 i$. Then vertex $\mathbf{T}_{1}=(1,0)=1+0 i$, while $\mathbf{T}_{2}=(1,1)=1+i$. Note that our function has $T(1)=1$ and $T(2)=1+i / \sqrt{1}=1+i$.

Now think of the multiplication of complex numbers: as Richard Feynman (1918 - 1988), an American theoretical physicist, observed famously, to a physicist complex multiplication is very simple: you multiply the magnitudes, and you add the angles. The angle of the complex number $1+i / \sqrt{n}$ has $\tan \phi_{n}=1 / \sqrt{n}$, which is exactly the central angle of the $n$th Theodorus triangle; and

\footnotetext{
${ }^{2}$ To use technology to gain insight and intuition, to visualize mathematical principles, to discover new relationships, and to test possibly false conjectures.

${ }^{3}$ Borwein never explicitly mentions the Spiral of Theodorus
} 
the magnitude of $T(2)$ is $|T(2)|=\sqrt{2}$ which is the length of the hypotenuse of the first Theodorus triangle (and the base of the second). Since $T(n+1)=(1+i / \sqrt{n}) T(n)$ we have

$$
\begin{aligned}
|T(n+1)| & =\sqrt{1+\frac{1}{n}}|T(n)| \\
& =\frac{\sqrt{n+1}}{\sqrt{n}}|T(n)|
\end{aligned}
$$

and since $|T(1)|=1$ we have by induction that $|T(n)|=\sqrt{n}$. Notice that this is exactly the length $\left|\overline{\mathrm{OT}_{n}}\right|$.

What we have just proved is that the complex curve $T(a)$ parameterized by the real parameter $a$ exactly interpolates the Theodorus spiral (provided, of course, that the infinite product defined when $a$ is not an integer actually exists; we don't do this here, because it demands knowledge of the convergence of infinite products which is not usually part of the standard curriculum at least until later; but the proof that it does exist, which is intelligible assuming the reader does have that background, is in [8]). We do, however, show that this works in Maple, in section 3. This analysis is very similar to Euler's interpolation of the gamma function, which indeed this is modelled on; see [5] or, better yet, the magnificent (Chauvenet prize-winning) paper [7].

Walter Gautschi, a Swiss-American mathematician, starts from here and writes in [9] the complex argument of $T(a)$ as the integral of a sum, so that

$$
T(a)=\sqrt{a} \exp \left(\frac{i}{2} \int_{\alpha=1}^{a} U(\alpha) d \alpha\right)
$$

with

$$
U(a)=\sum_{k=1}^{\infty} \frac{1}{(k+a-1)^{\frac{3}{2}}+\sqrt{k+a-1}} .
$$

As pointed out in [7] and in [9], this sum converges, but only "painfully slowly". Taking a million terms only gets you three significant figures of accuracy, if you work naively. This is also true of the Theodorus constant, defined as $U(1)$ or

$$
T=\sum_{k \geq 1} \frac{1}{k^{3 / 2}+k^{1 / 2}}
$$

...regarding its slope as it crosses the $0^{0}$ ray as a fundamental world constant and calling this world constant $T$ in honor of Theodorus, I wanted to determine $T$ to about eight or ten figures to the right of the decimal point.

-Philip J. Davis, [8, p. 40]

Davis then goes on to discuss two different methods to do so.

\section{Some experiments in Maple}

However, we can evaluate that very slowly-converging sum in Maple extremely simply. Everything is done for us, internally, by the Levin's $u$-transform [16]. [This is similar in effect to the first technique discussed by Davis, namely the use of the Euler-Maclaurin sum formula. But it's built-in, and works very well on this example.]

$$
\begin{gathered}
>U:=a \rightarrow \operatorname{evalf}\left(\operatorname{Sum}\left(\frac{1}{(k+a-1)^{\frac{3}{2}}+(k+a-1)^{\frac{1}{2}}}, k=1 . . \text { infinity }\right)\right) \\
U:=a \mapsto \text { evalf }\left(\sum_{k=1}^{\infty} \frac{1}{(k+a-1)^{\frac{3}{2}}+\sqrt{k+a-1}}\right)
\end{gathered}
$$


$>$ Digits := 100;

$$
\text { Digits }:=100
$$

$>$ CodeTools:-Usage $(U(1))$

memory used $=11.58 \mathrm{MiB}$, alloc change $=0$ bytes, cpu time $=62.00 \mathrm{~ms}$, real time $=48.00 \mathrm{~ms}$, gc time $=0 \mathrm{~ns}$

$$
\begin{aligned}
& 1.8600250792211903071806959157171433246665241215234 \\
& 51493049199503598342723399921320568838756499614496
\end{aligned}
$$

This gives us 100 digits of the Theodorus constant in about a twentieth of a second. But what we need to plot the spiral is really the integral

$$
\phi(a)=\frac{1}{2} \int_{\alpha=1}^{a} U(\alpha) d \alpha
$$

which gives the polar angle $\phi(a)$ of $T(a)=\sqrt{a} e^{i \phi(a)}$. While this is perfectly possible in Maple by using evalf/Int, and it is rapid and accurate for small values of $a$, as $a$ increases this takes increasingly more time to do. For $a=2$ using 15 Digits the computation takes less than 2 seconds, but for $a=16$ this takes about 8 seconds (on a 2021 vintage $1.3 \mathrm{GHz}$ Microsoft Surface Pro).

Gautschi goes on in [9] to find a definite integral expression for $\phi(a)$, which is both faster and more elegant than the integration of the accelerated sum above, in terms of special functions. In Maple's notation, this is $\phi(a)=G(a)$ where

$$
\begin{gathered}
>\text { epsilon }:=t \rightarrow \frac{t}{\exp (t)-1} \\
\epsilon:=t \mapsto \frac{t}{\mathrm{e}^{t}-1} \\
>G:=\text { alpha } \rightarrow \operatorname{Int}\left(\frac{t^{-\frac{1}{2}} \cdot \operatorname{epsilon}(t) \cdot(1-\exp (-(\operatorname{alpha}-1) \cdot t))}{t} \cdot \frac{\exp (-t)}{2 \cdot \operatorname{sqrt}(t)} \cdot \operatorname{erfi}(\operatorname{sqrt}(t)), t=0 . . \text { infinity }\right) \\
G:=\alpha \mapsto \int_{0}^{\infty} \frac{\epsilon(t) \cdot\left(1-\mathrm{e}^{-(\alpha-1) \cdot t}\right) \cdot\left(\frac{1}{2}\right) \cdot \mathrm{e}^{-t} \cdot \operatorname{erfi}(\sqrt{t})}{t^{\frac{3}{2}} \cdot \sqrt{t}} d t .
\end{gathered}
$$

This clever formulation involving the erfi function (also known as Dawson's Integral) is less computationally expensive to evaluate, for large $a$, than the integral of the accelerated sum above: while the sum takes about a fifth of a second to evaluate (at 15 Digits) at each value of $a$, the integral of $U(a)$ where $U(a)$ is evaluated by evalf/Sum takes about 8 seconds when $a=16$; whereas the integral of the special function takes only about 2 seconds. Having both methods available in Maple is good: the agreement of the two results leads to increased confidence.

However, a much faster way to evaluate

$$
G(a)=\phi(a)=\frac{1}{2} \int_{\alpha=1}^{a} U(\alpha) d \alpha
$$

for a succession of values, such as you might need to plot the continuous Spiral of Theodorus, is to solve the differential equation $\phi^{\prime}(a)=U(a) / 2$ numerically, over the range $1 \leq a \leq A$ where $A$ is the "return time" of the map, namely the value such that $\phi(A)=2 \pi$. This numerical solution takes about 2 seconds in Maple using the default rkf45 method, but thereafter the value of $\phi(a)$ is available essentially instantaneously for any $1 \leq a \leq A$ by evaluating the computed polynomial interpolant. This raises the question of how accurate some piecewise polynomial interpolant of the discrete spiral would be; that is a question we reserve for another day. 


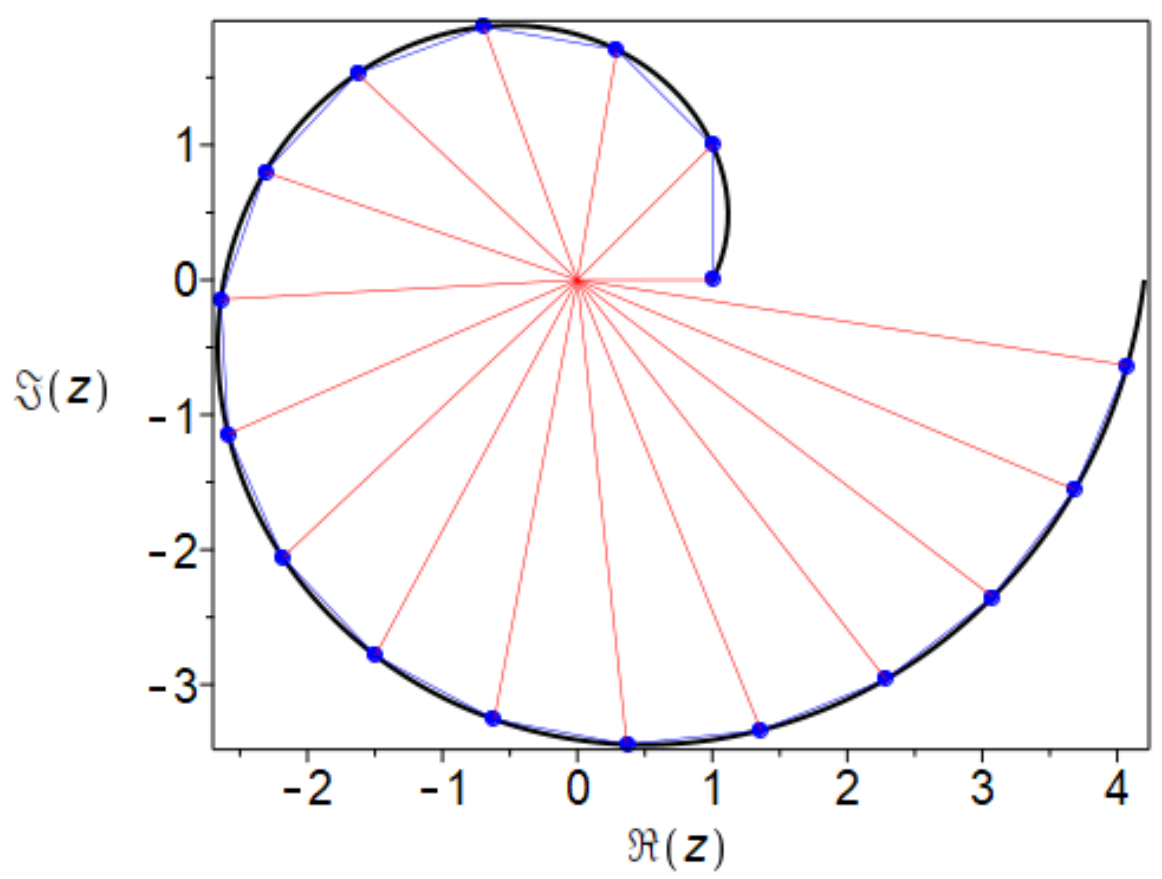

Fig. 3. The continuous version (black curved line) of the Spiral of Theodorus, plotted in Maple.

By use of fsolve, which takes about 5 minutes using the numerical quadrature of the evalf/Sum form of $U(a)$, we find $A=17.6459044714280$ to 15 Digits, with $\phi(A)=2 \pi$. This computation is much faster using the Gautschi definite integral, taking less than 30 seconds. The use of the numerical differential equation solver, with event handling, should take only about 2 seconds (essentially the same as just solving the system).

The computations in this section are available at This Maple Cloud Link.

\section{Experiments with physical triangles led to a surprise}

Gerofsky materialized the Spiral of Theodorus by creating various models of it from materials that included pieces of furniture, cardboard cutouts, and even edible pastry. [10]

During one of her workshops while re-arranging triangles used to build a model of the Spiral of Theodorus, Gerofsky placed the right triangles in reverse order, i.e. with the hypotenuse of the subsequent triangle set on the leg of the previous one, rather than on its hypotenuse as it the case with the Spiral of Theodorus. See Figure 4.

In this new arrangement of triangles, the vertices opposite to the leg of length 1 , annotated by $G_{0}, G_{1}, G_{2}, \ldots$ in Figure 5, form a spiral-like object which she called the reverse Wurzelschnecke. 

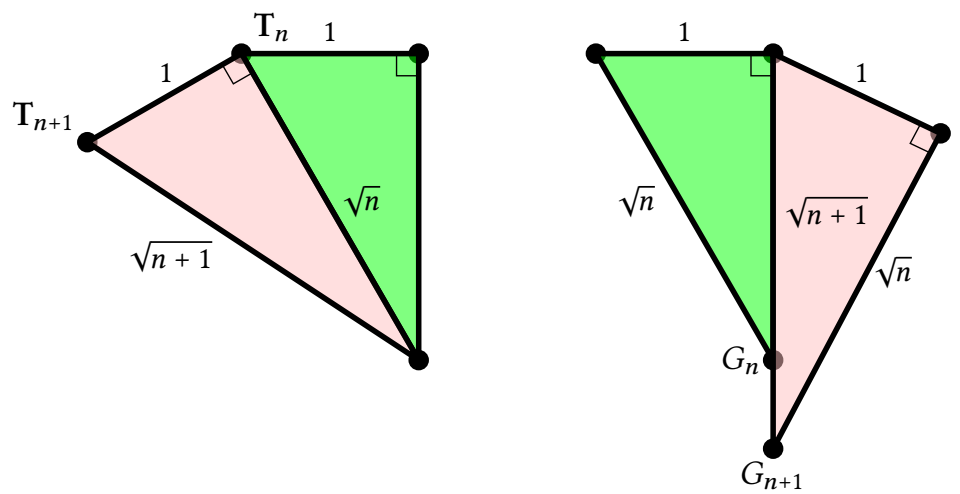

Fig. 4. Two characteristic adjacent right triangles in the Spiral of Theodourus (left) and the reverse Wurzelschnecke (right)

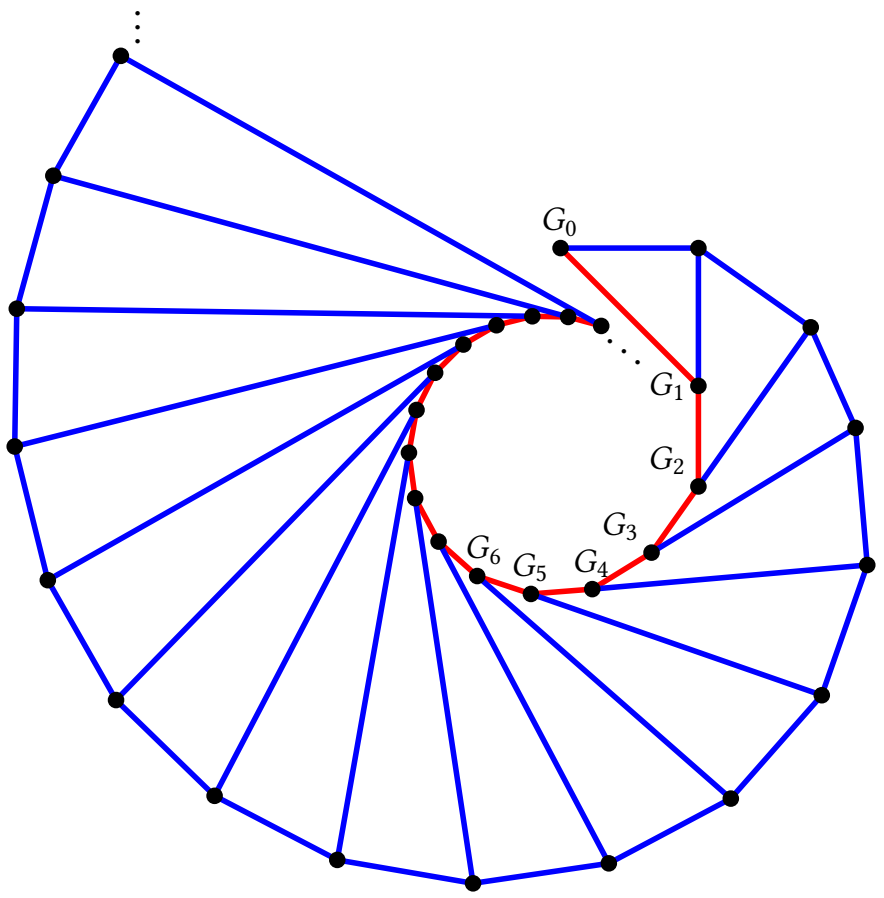

Fig. 5. Reverse Wurzelschnecke

In what follows we analyze some of the properties of the reverse Wurzelschnecke, both analytically and computationally. We also provide an elementary proof of the fact conjectured in [10], i.e. we prove that, when $n$ increases, the points $G_{n}$ approach the boundary of a certain circle. We determine the centre and the radius of that circle. 


\section{Reverse Wurzelschnecke}

In this section we draw the reverse Wurzelschnecke in the complex plane and denote its points by $z_{n}, n \in\{0,1,2, \ldots\}$. For $n \geq 1$ we denote the other two vertices of the $z_{n}$-determined characteristic triangle by $w_{n-1}$ and $w_{n}$. See Figure 6 .

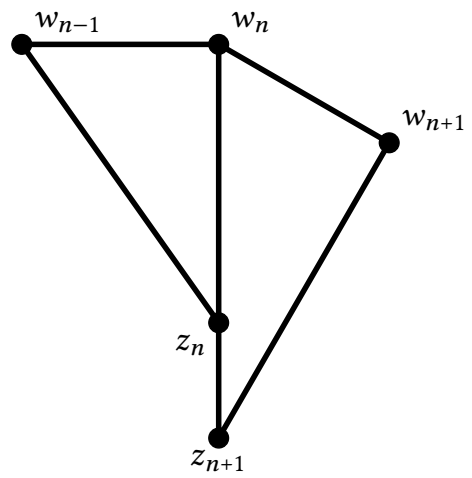

Fig. 6. Reverse Wurzelschnecke: Two characteristic adjacent right triangles in the complex plane

By construction,

$$
\left|w_{n}-z_{n}\right|=\sqrt{n},\left|w_{n}-w_{n+1}\right|=1,\left|w_{n+1}-z_{n+1}\right|=\sqrt{n+1}, \text { and }\left|w_{n}-z_{n+1}\right|=\sqrt{n+2} .
$$

In addition,

$$
\left|z_{n+1}-z_{n}\right|=\sqrt{n+2}-\sqrt{n} .
$$

Proposition 1. For all $n \in \mathbb{N}$,

$$
z_{n+1}=w_{n}+\sqrt{\frac{n+2}{n}} \cdot\left(z_{n}-w_{n}\right)
$$

Proof. Observe that $z_{n}, w_{n}$, and $z_{n+1}$ are three collinear points. This implies that there is $t>1$ such that

$$
z_{n+1}=w_{n}+t\left(z_{n}-w_{n}\right)
$$

Hence

Therefore

$$
t=\frac{z_{n+1}-w_{n}}{z_{n}-w_{n}} \Rightarrow|t|=\frac{\left|z_{n+1}-w_{n}\right|}{\left|z_{n}-w_{n}\right|}=\sqrt{\frac{n+2}{n}} .
$$

$$
z_{n+1}=w_{n}+\sqrt{\frac{n+2}{n}} \cdot\left(z_{n}-w_{n}\right)
$$

\section{It is a circle!}

We construct the Spiral of Theodorus, $\mathbf{T}_{1}, \mathbf{T}_{2}, \mathbf{T}_{3}, \ldots$, with the initial point $\mathbf{O}$. Recall that by construction

$$
\left|\overline{\mathrm{OT}_{n}}\right|=\sqrt{n},\left|\overline{\mathrm{T}_{n} \mathrm{~T}_{n+1}}\right|=1, \overline{\mathrm{OT}_{n}} \perp \overline{\mathrm{T}_{n} \mathrm{~T}_{n+1}} .
$$

Through each point $\mathbf{T}_{n+1}$ we construct the line $t_{n}, t_{n} \perp \overline{\mathbf{T}_{n} \mathbf{T}_{n+1}}$. Let

$$
\mathrm{S}_{n}=t_{n} \cap t_{n+1} \text {. }
$$

See Figure 7. 


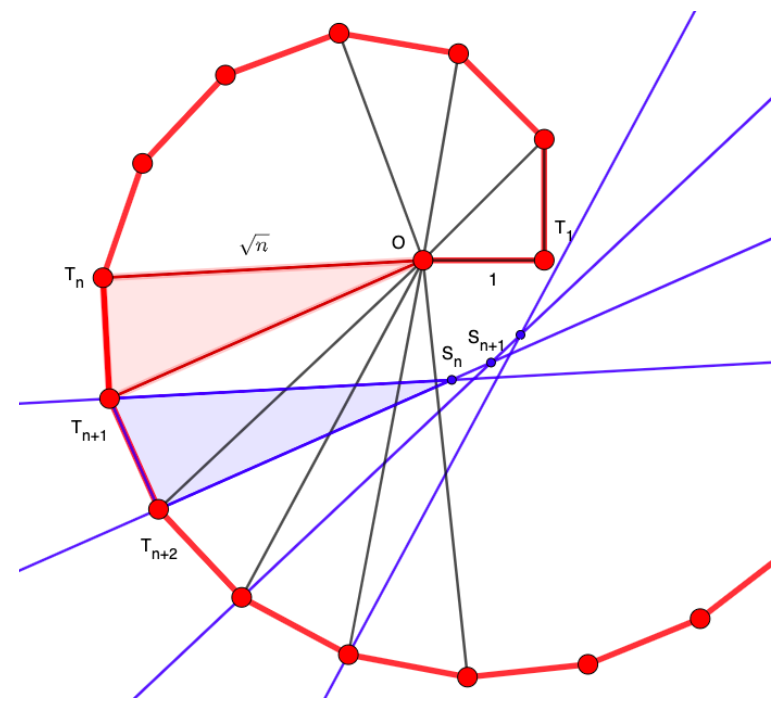

Fig. 7. Two Spirals

Proposition 2. The sequence of points $\left\{\mathrm{S}_{n}\right\}_{n \in \mathbb{N}}$ form the reverse Wurzelschnecke.

Proof. Observe that

$$
\overline{\mathrm{OT}_{n+1}} \perp \overline{\mathrm{T}_{n+1} \mathrm{~T}_{n+2}} \text { and } \overline{\mathrm{S}_{n} \mathrm{~T}_{n+1}} \perp \overline{\mathrm{T}_{n} \mathrm{~T}_{n+1}} .
$$

This means that $\angle \mathbf{T}_{n} \mathbf{T}_{n+1} \mathbf{O}$ and $\angle \mathbf{T}_{n+2} \mathbf{T}_{n+1} \mathrm{~S}_{n}$ are congruent, as two angles with two pairs of mutually perpendicular rays.

It follows that the right triangles $\Delta \mathbf{T}_{n} \mathbf{T}_{n+1} \mathbf{O}$ and $\Delta \mathbf{T}_{n+2} \mathbf{T}_{n+1} \mathrm{~S}_{n}$ are congruent. In particular

$$
\left|\overline{\mathrm{S}_{n} \mathrm{~T}_{n+2}}\right|=\left|\overline{\mathrm{OT}_{n}}\right|=\sqrt{n} \text {, and }\left|\overline{\mathrm{S}_{n} \mathrm{~T}_{n+1}}\right|=\left|\overline{\mathrm{OT}_{n+1}}\right|=\sqrt{n+1} \text {. }
$$

Also, observe that, by construction, the points $\mathrm{T}_{n+2}, \mathrm{~S}_{n}, \mathrm{~S}_{n+1}$ are collinear.

Therfore, the points $S_{1}, S_{2}, S_{3}, \ldots$ form the reverse Wurzelschnecke.

Proposition 3. For $\mathrm{O}$ and the sequence $\left\{\mathrm{S}_{n}\right\}_{n \in \mathbb{N}}$ as depicted in Figure 7,

$$
\lim _{n \rightarrow \infty}\left|\overline{\mathrm{OS}_{n}}\right|=1 \text {. }
$$

Proof. We refer to Figures 7 and 8.

We consider the trapezoid $\mathrm{OT}_{n} \mathrm{~T}_{n+1} \mathrm{~S}_{n}$. We observe that

$$
\left|\overline{\mathrm{OT}_{n}}\right|=\sqrt{n},\left|\overline{\mathrm{S}_{n} \mathrm{~T}_{n+1}}\right|=\sqrt{n+1},\left|\overline{\mathbf{T}_{n} \mathbf{T}_{n+1}}\right|=1
$$

and that

It follows that

$$
\angle \mathbf{T}_{n+1} \mathbf{T}_{n} \mathbf{O}=\angle \mathbf{T}_{n} \mathbf{T}_{n+1} \mathrm{~S}_{n}=\frac{\pi}{2}
$$

$$
\begin{aligned}
\lim _{n \rightarrow \infty}\left|\overline{\mathrm{OS}_{n}}\right|^{2} & =\lim _{n \rightarrow \infty}\left((\sqrt{n+1}-\sqrt{n})^{2}+1\right)=\lim _{n \rightarrow \infty}\left(\left(\frac{(\sqrt{n+1}-\sqrt{n})(\sqrt{n+1}+\sqrt{n})}{\sqrt{n+1}+\sqrt{n}}\right)^{2}+1\right) \\
& =\lim _{n \rightarrow \infty}\left(\left(\frac{1}{\sqrt{n+1}+\sqrt{n}}\right)^{2}+1\right) \\
& =1 .
\end{aligned}
$$




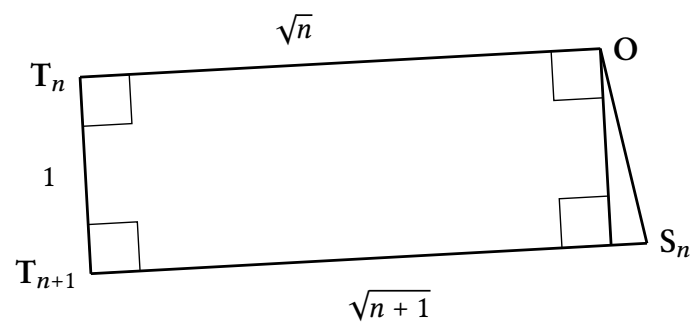

Fig. 8. The trapezoid. The lower line $\mathrm{T}_{n+1} \mathrm{~S}_{n}$ is length $\sqrt{n+1}$ while the upper line $\mathrm{T}_{n} \mathrm{O}$ is length $\sqrt{n}$.

Indeed, the approach to 1 is monotonic, from above. By Maple, we have $>(\operatorname{sqrt}(n+1)-\operatorname{sqrt}(n))^{2}+1$

$$
(\sqrt{n+1}-\sqrt{n})^{2}+1
$$

$>\operatorname{asympt}(\operatorname{sqrt}((\mathbf{1 5})), n)$

$$
1+\frac{1}{8 n}-\frac{9}{128 n^{2}}+\frac{49}{1024 n^{3}}-\frac{1173}{32768 n^{4}}+\frac{7431}{262144 n^{5}}+\mathrm{O}\left(\frac{1}{n^{6}}\right)
$$

Theorem 1. Points $\mathrm{S}_{1}, \mathrm{~S}_{2}, \mathrm{~S}_{3}, \ldots$ approach the circle with the centre at $\mathrm{O}$ and radius 1 .

Proof. The claim follows from Proposition 3.

Proposition 4. The angles $\psi_{n} \bmod 2 \pi$ of the points $\mathrm{S}_{n}$ on the inner curve of the reverse spiral are asymptotically equidistributed in the interval $[0,2 \pi)$.

Proof. The angles of the spiral of Theodorus, say $\theta_{n}=\sum_{k=2}^{n} \phi_{k}$ where $\phi_{n}=\arctan (1 / \sqrt{n})$ are the internal angles of the triangles, are known to be equidistributed by a theorem established by Edmund Hlawka (1916 - 2009), an Austrian mathematician, in 1980 [8, Historical Supplement $\mathrm{H}]$. Consider the isoceles triangle $\mathbf{O T}_{n+1} \mathrm{~S}_{n}$ in Figure 8. The angle $\angle \mathrm{OS}_{n} \mathbf{T}_{n}$ is $\left(\pi-\phi_{n+1}\right) / 2$, so the angle $\angle \mathbf{T}_{n} \mathrm{OS}_{n}$ is asymptotically $\pi / 2+O(1 / \sqrt{n})$ more than the angle $\theta_{n}$ of $\mathbf{T}_{n}$, as $n \rightarrow \infty$. This asymptotically constant shift does not affect the equidistribution: since the $\theta_{n}$ are equidistributed, so are the $\psi_{n}$.

\section{Code Implementation}

The Maple code used for the creation of Figure 3 did not have to go very far; only 17 nodes were needed. But if one wants to compute many triangles (as we did before we discovered that the inner curve approached a circle), a faster code is needed. Indeed, it was written first, and in Python.

The code written in Python can calculate and plot the reverse Wurzelschnecke, as well as show how it overlaps with the Spiral of Theodorus. It can be found on Ewan Brinkman's GitHub here.

\section{Running the Code}

All of the required packages are listed in the file requirements. txt. Python version 3 should be used. When run, two options will appear in the terminal. Note that whenever the program ask for input, pressing the enter key will select a default option. 
To plot data, type 1 and press enter. The program will look for a data file with the name DATA_FILE (set in settings.py), in the data folder. Options when plotting are shown in Section 7. Figure 9 shows an example plot of the first 100 triangles.

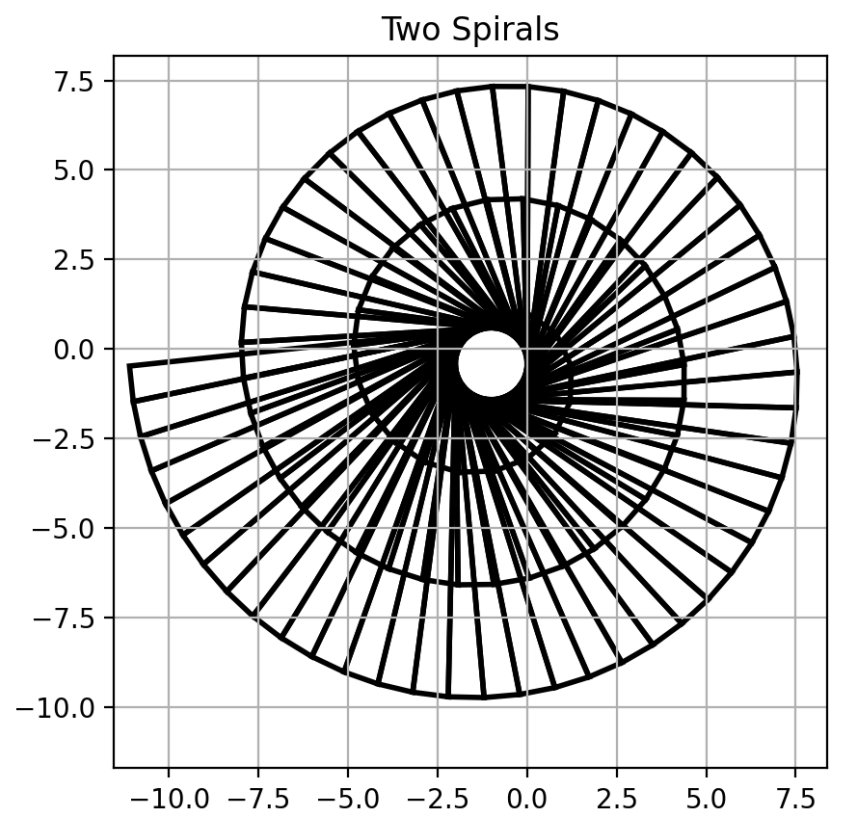

Fig. 9. First 100 triangles of the reverse Wurzelschnecke.

To calculate plotting data, type 2 and press enter. Next, input how many triangles should be calculated. A negative will calculate triangles forever, until the program is interrupted. Finally, enter how often it should save calculated triangles. The default, 1, saves every triangle. A value of 2 will save every other triangle, while entering 1000 will save every thousandth triangle. When saving the calculated data, the program will look for a data file with the name DATA_FILE (set in settings.py), in the data folder. If no file is found, a new file with the name DATA_FILE will be created. Options for calculating triangles are shown in Section 7.

\section{The Algorithm}

Each triangle is calculated using a previous triangle's data. Given an $n$th triangle, the program will use that triangle's outside right vertex, rotation and triangle number plus 1 to calculate the next triangle. For example, given the second triangle, the program will use its outside right vertex of $\left(\frac{\sqrt{6}}{3}, 1-\frac{\sqrt{3}}{3}\right)$, its rotation of $\arctan \frac{\sqrt{2}}{2}$, and a triangle number of 3 .

First, the new triangle's inside leg length is calculated by taking the square root of the triangle number. See 7 for using a custom function for calculating triangle side lengths. Next, the new triangle is connected to the previous triangle, without being rotated. Figure 10 shows what the third triangle would look like at this stage.

Next, the outside right vertex and inside vertex of the triangle are rotated clockwise around the vertex connected to the previous triangle. The rotation is the previous triangle's rotation, plus 


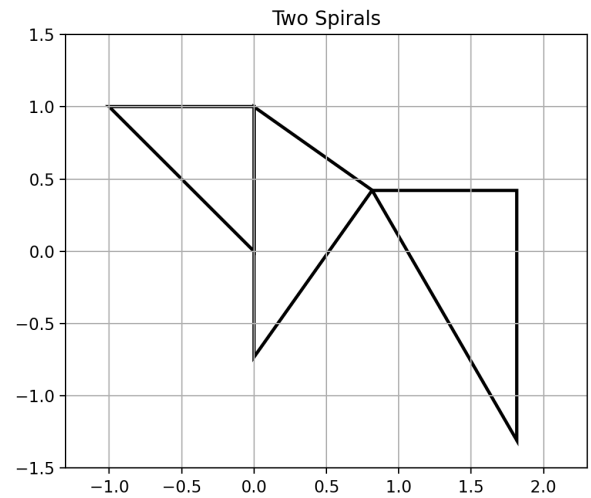

Fig. 10. The third triangle before being rotated.

$\arctan \frac{\text { outside leg }}{\text { inside leg }}$ of this new triangle. Figure 11 shows what the third triangle would now look like.

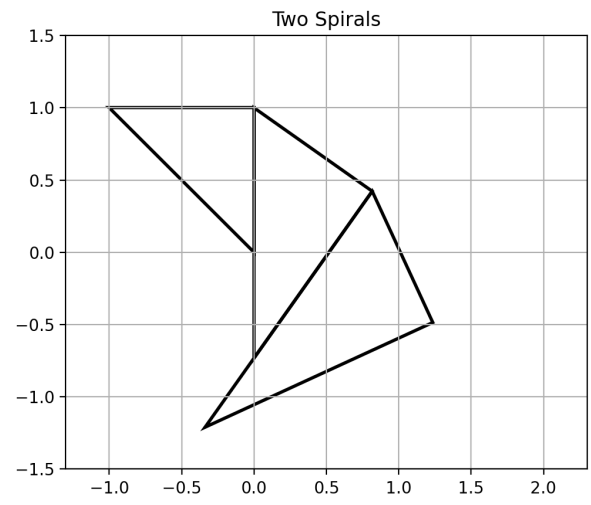

Fig. 11. The third triangle after being rotated.

This new rotation value will then be passed onto the next triangle, as well as the triangle's outside right vertex and one more than its triangle number.

Each triangle, an instance of the class Triangle from the file triangles.py, performs these calculations using the class method calculate_triangle.

\section{Settings}

The constants below are located in the file settings.py.

Settings for plotting: 
- SHOW_TRIANGLES: set to True to plot the reverse Wurzelschnecke in full. Set it to False to only plot a single vertex of each triangle. The vertex to be plotted can be set using PLOT_TRIANGLE_POINT (see below).

- PLOT_TRIANGLE_POINT: if SHOW_TRIANGLES is False, this determines which vertex of the triangles is plotted. The options are: "inside" for the inner vertices which form the circle, or either "outside_left" or "outside_right" for the other triangles' vertices.

- ANIMATE_SPIRAL_OF_THEODORUS: set to True to play an animation showing how the Spiral of Theodorus overlaps with the reverse Wurzelschnecke. See more in the section 7.

- SPIRAL_OF_THEODORUS_AMOUNT: how many triangles should be used during the animation showing how the Spiral of Theodorus overlaps with the Reverse Wurzelschnecke. Set the parameter ANIMATE_SPIRAL_OF_THEODORUS to True to play the animation.

- SHOW_CIRCLE: set to True to plot the circle that the inside vertices of the triangle go around, as well as the circle's center.

- ANIMATE_PLOT: if set to True, each triangle of the reverse Wurzelschnecke will be plotted with a delay in between each one. The delay can be set with ANIMATION_INTERVAL.

- ANIMATION_INTERVAL: the amount of milliseconds to pause between each triangle while plotting. This requires ANIMATE_PLOT to be True.

- CONNECT_POINTS: set to True to connect each triangle's vertex to the next with a straight line while plotting. SHOW_TRIANGLES should be False and a vertex should be set with the variable PLOT_TRIANGLE_POINT.

- PLOT_TITLE: a string used as the title of the plot.

- COLOUR_PERCENT_DONE: set to True to colour the triangles or triangle vertices based on how many have been plotted before them. For example, if the first 10 triangles are plotted, triangles closer to 1 will be closer to one side of the colour gradient, while triangles closer to 10 will be closer to the other side of the colour gradient.

Settings for calculating triangles:

- EXACT_VALUES: set to True to use exact values instead of decimal approximations during calculations. Exact values are calculated using sympy. Note, using exact values becomes slower as the number of triangles increases. It is recommended to set this to False.

- OUTSIDE_LEG_LENGTH: the length of the triangles' outside legs. If wanted, this can be changed to a value other than 1.

- CUSTOM_HYPOTENUSE_FUNCTION: set to True to use a custom function for calculating the hypotenuse of each triangle. The function is called calculate_hypotenuse and can be found in the file utils.py. For example, instead of taking the square root of the triangle's number plus 1, a cube root could be done instead. The function takes the triangle's number as input (triangle number 1 , triangle number 2 , etc.), and must return the hypotenuse of the triangle. The triangle's inside leg is then calculated using the hypotenuse. Note, the outside leg length can be set using OUTSIDE_LEG_LENGTH.

Settings for the triangle data file:

- DATA_FILE: the name of the data file for reading and writing calculated triangles.

- HEADERS: the headers of the csv file which stores the calculated triangles.

\section{Visualizing the Two Spirals Together}

In the file settings.py, setting the constant ANIMATE_SPIRAL_OF_THEODORUS to True will play an animation showing how the Spiral of Theodorus (the red lines) can be transformed to overlap with 
the Reverse Wurzelschnecke (the black triangles). The constant SPIRAL_OF_THEODORUS_AMOUNT sets how many triangles should be used for the Spiral of Theodorus. A copy of the animation can also be found on Ewan Brinkman's GitHub here.

During the animation, the reverse Wurzelschnecke does not move. Instead, the Spiral of Theodorus begins by being reflected about the line $x=1$. Next, it is rotated $\arctan 1$ radians around the point $(1,1)$, before being translated 2 units left.

\section{Large Calculations}

When billions of triangles are calculated with this program, interesting behaviour, likely numerical in origin, appears. Figure 12 shows the inside vertices of every one thousandth triangle from the 12.41 billionth triangle to the 12.415 billionth triangle. The vertices are coloured on a gradient from black to red, where black points are closer to the 12.41 billionth triangle and red points are closer to the 12.415 billionth triangle. The shaded red area represents the circle the triangles circle around, with the pink point being that circle's centre.

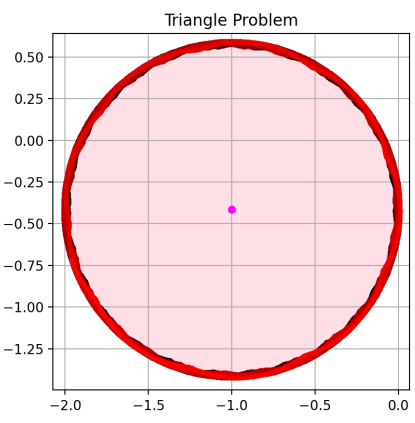

(a) Every inside vertex

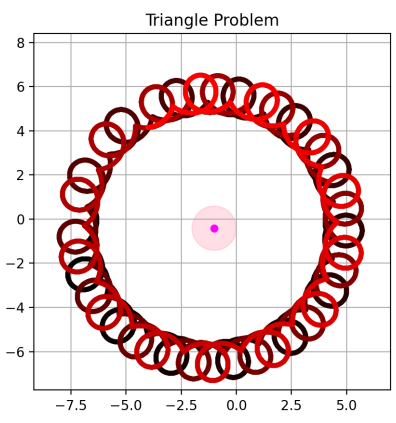

(b) Every 3000th inside vertex

Fig. 12. a) Inside vertices of triangles near 12.41 billion. b) Inside vertices of every three thousandth triangle from 50 billion to 50.05 billion.

It appears that the inside vertices have begun to wobble slightly. After more triangles are calculated, this became more apparent. Furthermore, the path the inside vertices trace appear to be shifting away from the centre of the circle that the inside vertices were originally going around. Figures 13 to 12(b) show some examples. In Figure 12(b), the inside vertices have even moved away from the circle. [Note: one hypothesis is floating point error. We reran the program multiple times to see if the results were reproducible. This just showed that the results were deterministic; but floating-point error is not random, so that did not rule out rounding errors. For triangle number $10^{8}$, the side length is $10^{4}$; even using single precision, we would expect a side length accurate to $10^{-3}$ which would look exact to the human eye. If floating-point error is significant here, it must be because of accumulated errors in the summation of the angles. We have not yet investigated this in detail. ]

In Figure 15 we plot the relative differences $\left|S_{n, \text { Python }}-S_{n, \text { Maple }}\right| /\left|S_{n, \text { Maple }}\right|$ for $n=10,100, \ldots, 10^{11}$ (one hundred billion, in the North American sense which we have used consistently throughout this paper). We see a growth in these differences which we have visually fit with a curve $C \cdot n^{1.5}$, with $C$ a constant. We view this as evidence for cumulative rounding errors in the Python program, which 


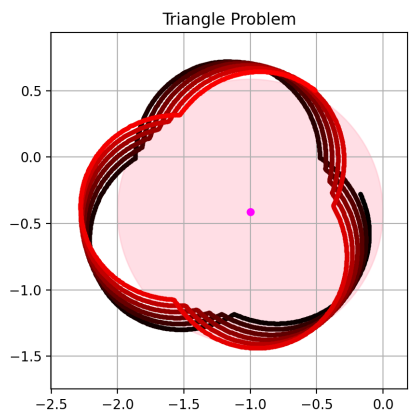

(a) From 18.33billion

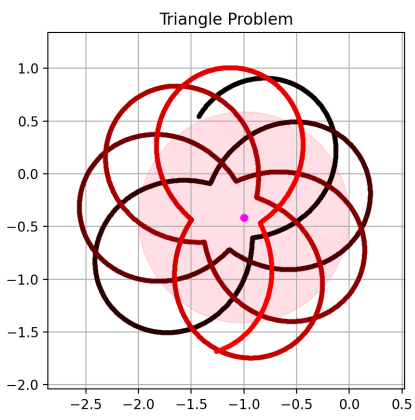

(b) From 30.25 billion

Fig. 13. a) Inside vertices of every one thousandth triangle from 18.33 billion to 18.335 billion. b) Inside vertices of every one thousandth triangle from 30.25 billion to 30.255 billion.

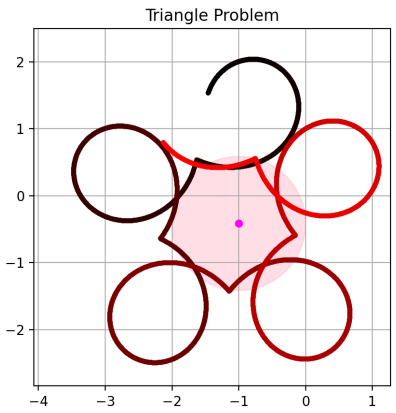

(a) Every thousandth

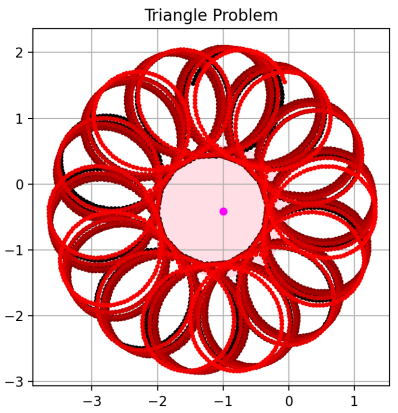

(b) Every ten thousandth

Fig. 14. a) Inside vertices of every one thousandth triangle from 42.36 billion to 42.365 billion. b) Inside vertices of every ten thousandth triangle from 42.36 billion to 42.46 billion.

does more computation than the Maple computation (which indeed uses sequence acceleration to avoid computation).

\section{Concluding Remarks}

This exploration began with an observation from physical triangles put together in a novel way; then Ewan Brinkman wrote the Python program to compute a large number of triangles, using real arithmetic and trigonometry to put them together. The first puzzle this computation generated was "what is the true shape of the curve being traced out in the centre?" The Python program gave us important clues. Following up on those clues, we were able to give a proof that the ultimate shape is indeed a circle (contradicting the large-scale experiments with the Python program, and thus demonstrating that the interesting behaviour shown must be numerical in origin). The points approach the circle monotonically in radius with the radius of the $n$th point $\left|\mathrm{S}_{n}\right|=1+1 /(8 n)+O\left(1 / n^{2}\right)$. We also showed that the points are equidistributed on that circle, again contradicting some of the 


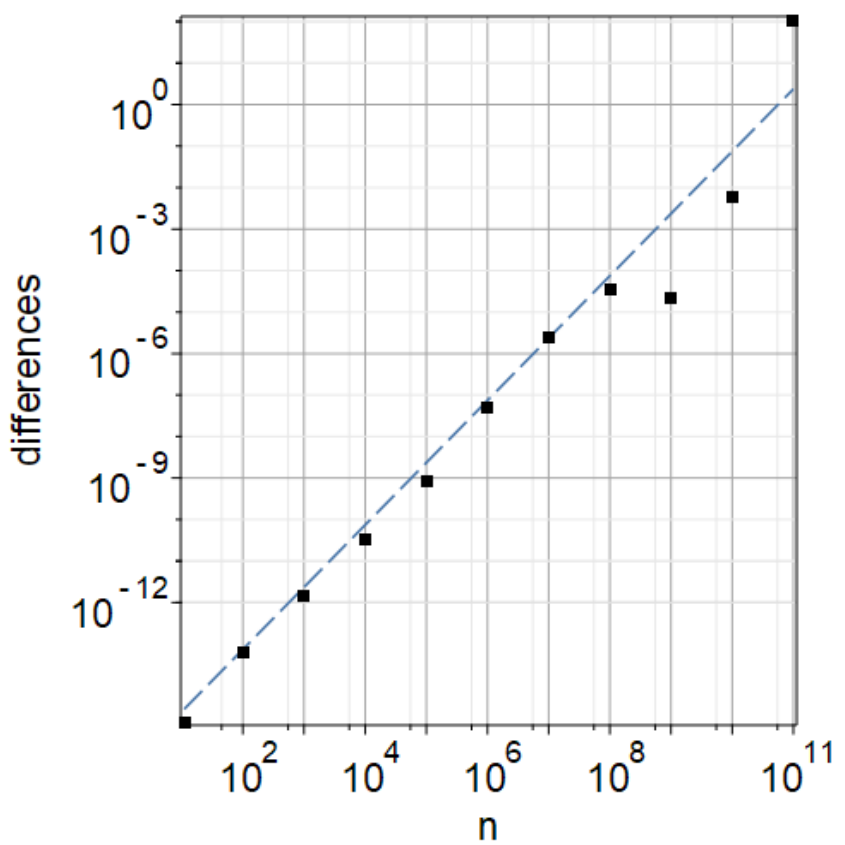

Fig. 15. Relative magnitude of the differences between $\mathrm{S}_{n}$ computed in Python and in Maple for $n=10,100$, $\ldots, 10^{11}$. The dashed line shows growth like $O\left(n^{1.5}\right)$.

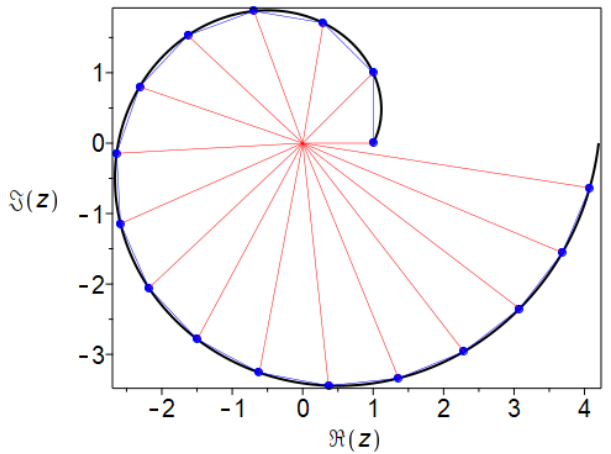

(a) Forward

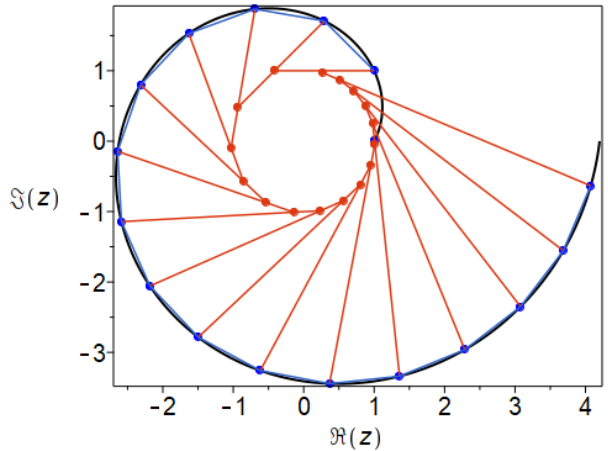

(b) Reverse

Fig. 16. Comparing the two spirals, using the same orientation for simplicity. The fact that all $\mathrm{S}_{n}$ lie outside the unit circle is not quite evident in Figure 16(b) above, but it is true. 
very large computations carried out with the Python program. This suggests that the detailed behaviour there might be of interest to numerical analysts; in Figure 12(b) we see something like "epicycles," for instance; we conclude that these might be accumulated rounding errors. We suspect that even in spite of the visible regularity of the deviations that this is the case.

Computation of the location of the $n$th point $\mathbf{T}_{n}$ on the Spiral of Theodorus, even for $n$ very large, is possible in Maple by use of Levin's $u$-transform to accelerate the summation of angles. Computing the trillionth $\mathrm{T}_{n}$ takes only milliseconds. To 35 significant figures, computed using 50 Digits in Maple (which took only 192 milliseconds), the trillionth point on the spiral of Theodorus is $\mathbf{T}_{10^{12}}=-964121.74674046601468450879463366548-265460.46308652576745796389309002521 i$. Similarly, the location of the $n$th point $S_{n}$ on the inner curve, approaching the circle, is likewise quickly computable. The corresponding trillionth inner point $\mathrm{S}_{n}$, again computed by sequence acceleration in only 28 milliseconds, is $\mathrm{S}_{10^{12}}=0.26545998102565239710444133249300938$ $0.96412187947069755798057508150144433 i$. Whether or not the sequence acceleration technique in use (Levin's $u$-transform) is giving us faulty information or not we leave for future investigation Preliminary experiments comparing with other techniques such as Euler-Maclaurin summation have not shown any contradiction so far, however.

After performing the initial Python experiments, we connected with the material of Philip J. Davis' lovely book [8], some of which we summarized in section 2 . We then confirmed some of those results by use of Maple in Section 3. See also [11] and [6]. Still, there seems lots left to explore. A good place to start would be [8, Supplement B], written by Arieh Iserles, a professor of numerical analysis at Cambridge University with extensive interests including dynamical systems; his supplement to Davis' book generalizes the Theodorus spiral in several ways, including to matrix iterations.

\section{References}

[1] Jonathan M. Borwein. Aesthetics for the working mathematician. In Mathematics and the Aesthetic, pages 21-40. Springer, 2006.

[2] Jonathan M. Borwein. Implications of experimental mathematics for the philosophy of mathematics. Proof and other dilemmas: Mathematics and philosophy, pages 33-60, 2008.

[3] Jonathan M. Borwein. The life of modern Homo habilis mathematicus: Experimental computation and visual theorems. In Tools and Mathematics, pages 23-90. Springer, 2016.

[4] Jonathan M. Borwein and David H. Bailey. Mathematics by experiment : plausible reasoning in the 21st century. AK Peters, Wellesley, Mass, 2nd edition, 2008.

[5] Jonathan M. Borwein and Robert M. Corless. Gamma and factorial in the Monthly. American Mathematical Monthly, 125(5):400-424, 2018.

[6] David Brink. The Spiral of Theodorus and sums of zeta-values at the half-integers. The American Mathematical Monthly, 119(9):779, 2012.

[7] Philip J. Davis. Leonhard Euler's integral: A historical profile of the gamma function. The American Mathematical Monthly, 66(10):849-869, 1959.

[8] Philip J. Davis. Spirals: From Theodorus to Chaos. AK Peters, Wellesley, Massachussetts, 1993.

[9] Walter Gautschi. The spiral of Theodorus, numerical analysis, and special functions. Journal of computational and applied mathematics, 235(4):1042-1052, 2010.

[10] Susan Gerofsky, S Brackett Robertson, and Veselin Jungić. Exploring the Wurzelschnecke: Learning geometry, number and design with the Spiral of Theodorus. In Bridges Conference Proceedings, pages 383-390. Tessellations Publ, 2021.

[11] Detlef Gronau. The spiral of Theodorus. The American Mathematical Monthly, 111(3):230-237, 2004.

[12] Thomas Heath. A history of Greek mathematics. Dover Publications, New York, 1981.

[13] Veselin Jungić and Andrijana Burazin. On experimental mathematics and mathematics education. American Mathematical Monthly, 128(9):832-844, 2021.

[14] John J. O'Connor and Edmund F. Robertson. Theodorus of Cyrene, 1999.

[15] Bernhard Rietzl. Nautilus theodori, 2016. http://gallery.bridgesmathart.org/exhibitions/2016-bridges-conference/ bernhard-rietzl.

[16] Ernst J. Weniger. Nonlinear sequence transformations for the acceleration of convergence and the summation of divergent series. Computer Physics Reports, 10(5-6):189-371, December 1989. 